\title{
Pulse Photoplethysmography Derived Respiration for Obstructive Sleep Apnea Detection
}

\author{
Jesús Lázaro ${ }^{1,2}$, Eduardo Gil ${ }^{3,4}$, Margot Deviaene ${ }^{1,2}$, Raquel Bailón ${ }^{3,4}$, \\ Dries Testelmans ${ }^{5}$, Bertien Buyse ${ }^{5}$, Carolina Varón ${ }^{1,2}$, Sabine Van Huffel ${ }^{1,2}$ \\ ${ }^{1}$ Department of Electrical Engineering (ESAT), STADIUS Centre for Dynamical Systems, Signal \\ Processing and Data Analytics, KU Leuven, Leuven, Belgium \\ ${ }^{2}$ IMEC, Leuven, Belgium \\ ${ }^{3}$ BSICoS Group, Aragón Institute for Engineering Research (I3A), IIS Aragón, University of \\ Zaragoza, Zaragoza, Spain \\ ${ }^{4}$ CIBER in Bioengineering, Biomaterials and Nanomedicine (CIBER-BBN), Madrid, Spain \\ ${ }^{5}$ University Hospital Leuven (UZ Leuven), Leuven, Belgium
}

\begin{abstract}
Five time series which are known to be modulated by respiration are derived from the pulse photoplethysmographic (PPG) signal, and they are analyzed for obstructive sleep apnea (OSA) detection: Pulse rate, amplitude, and width variabilities ( $P R V, P A V$, and $P W V$, respectively), pulse upslopes, and slope transit time (STT). A total of 26 polysomnographic recordings were split in 1-min segments which were manually labeled as OSA (653 segments), normal breathing (7204 segments), or other pulmonary events. For each one of the 5 PPG-derived series, 4 features were extracted: the standard deviation, the power at high and low frequency (PLF) bands, and the normalized PLF. These 20 features were used as input of a least-squares support vector machine classifier using an RBF kernel. Results show an accuracy of $72.66 \%$, suggesting that the analyzed features are promising for the detection of OSA from only the PPG signal.
\end{abstract}

\section{Introduction}

Obstructive sleep apnea (OSA) syndrome remains the most common type of sleep-disordered breathing. It consists of repetitive periods of upper airway occlussion during sleep interrupting the airflow to the lungs. An arousal is generated by the autonomic nervous system (ANS) in order to restore respiration, but at the same time, the arousal disturbs the sleep. These episodes may occur hundreds of times in a single night having severe health implications including hypersomnolence, excessive daytime sleepiness, insomnia, nocturia, memory loss, attention deficit, and depression [1]. Moreover, OSA symdrome is associated with an increased risk of cardiovascular events such as coronary artery disease, myocardial infarction, and stroke [2].

The diagnosis of OSA syndrome in adults is based on a complete overnight recording known as polysomnography or polygraphy, depending on the recording of the electroencephalogram or not. However, it remains a very expensive procedure as it requires specialized equipment, expert personnel, and many sensors reducing the comfort of the patient and affecting physiological sleep.

Different alternatives have been proposed, aimed to reduce costs, invasiveness, and/or to make the process more convenient for ambulatory scenarios. Some of these alternatives are based on the electrocardiogram (ECG), often combining features based on heart rate variability (HRV) for quantifying the ANS dynamics with other features related to respiration [1]. Other alternatives are based on the pulse photoplethysmographic (PPG) signal which also offers ANS information through pulse rate variability (PRV) and it is also affected by some respiratory modulations [3]. The PPG signal can be recorded by a pulse oximeter which is widely used in the clinical routine for measuring the peripheral oxygen saturation $\left(\mathrm{SpO}_{2}\right)$ while being a simple, low-price, and comfortable sensor and therefore particularly interesting for sleep studies. Because of the above mentioned issues, the PPG-based methods for OSA detection usually use also features extracted from $\mathrm{SpO}_{2}$. However, not all apnea events lead to an oxygen desaturation event, so some methods based only on PPG signal have also been proposed [4-6].

Pulse rate, amplitude, and width variability (PRV, PAV, and PWV, respectively) are 3 series derived from the PPG signal which have been studied for respiratory rate estimation [3]. Other PPG series which have been related to 
respiration are the pulse upslope [7] and the slope transit time (STT) [8]. In this paper, the potential for discriminating OSA from normal breathing (NB) of PRV, PAV, PWV, pulse upslope, and STT is studied.

\section{Methods}

\subsection{Data and signal preprocessing}

A data set containing 26 polysomnographic recordings performed at University Hospital Leuven (UZ Leuven) was used. These recordings included PPG signals recorded from index finger at a sampling rate of $f_{s}=500 \mathrm{~Hz}$ by a Nonin WristOx2 3150. Respiratory events were annotated by experienced personnel of UZ Leuven according to the 2012 American Academy of Sleep Medicine criteria $\left(\mathrm{AASM}_{12}\right)$ [9]. As in [1], recordings were split in 1-min segments which were labeled as OSA, NB, or other pulmonary events based on the previously mentioned annotations. Only OSA and NB segments were analyzed in this study.

For PPG preprocessing, a low-pass filter with a cut-off frequency of $35 \mathrm{~Hz}$ was applied. For the $i^{\text {th }} \mathrm{PPG}$ pulse, the apex point $\left(n_{\mathrm{A}_{i}}\right)$ was detected by an algorithm based on a low-pass derivative filter and a time-varying threshold [5]. Basal point $\left(n_{\mathrm{B}_{i}}\right)$ which corresponds to the minimum previous to $n_{\mathrm{A}_{i}}$, and medium point $\left(n_{\mathrm{M}_{i}}\right)$ defined as the time instant when PPG pulse reaches half of its amplitude, were also automatically determined as in [5]. Furthermore, the onset $\left(n_{\mathrm{O}_{i}}\right)$ and end points $\left(n_{\mathrm{E}_{i}}\right)$ were detected by an algorithm based on the first derivative [3].

\subsection{PPG-derived series}

Five series which have been previously related to respiration were extracted from PPG signal: PRV, PAV, PWV, pulse upslopes, and STT. The first 3 series were extracted as in [3]: PRV was generated by the inverse interval function using $n_{\mathrm{M}_{i}}$ as fiducial point, PAV was computed using $n_{\mathrm{B}_{i}}$ as reference, and PWV was computed as the time between $n_{\mathrm{O}_{i}}$ and $n_{\mathrm{E}_{i}}$.

For the STT measurement, $n_{\mathrm{O}_{i}}$ was considered the onset of the pulse upslope. The end of the pulse upslope $\left(n_{\mathrm{SE}_{\mathrm{E}}}\right)$ was detected by using a similar algorithm. Let $x(n)$ be the PPG signal, and $x^{\prime}(n)$ its 5-Hz-lowpass derivative version. Then, the maximum upslope point $\left(n_{\mathrm{U}_{i}}\right)$ is set as the absolute maximum of $x^{\prime}(n)$ within the $300 \mathrm{~ms}$ prior to $n_{\mathrm{A}_{i}}$. Next, $n_{\mathrm{S}_{\mathrm{E} i}}$ is set at that point where $x^{\prime}(n)$ falls to a percentage $\eta=0.3$ of its value at $n_{\mathrm{U}_{i}}$.

Subsequently, STT was computed as the time interval between $n_{\mathrm{O}_{i}}$ and $n_{\mathrm{S}_{\mathrm{E} i}}$. An illustration of STT definition is given in Fig. 1. On the other hand, the pulse upslope was set as $x^{\prime}\left(n_{\mathrm{U}_{i}}\right)$.

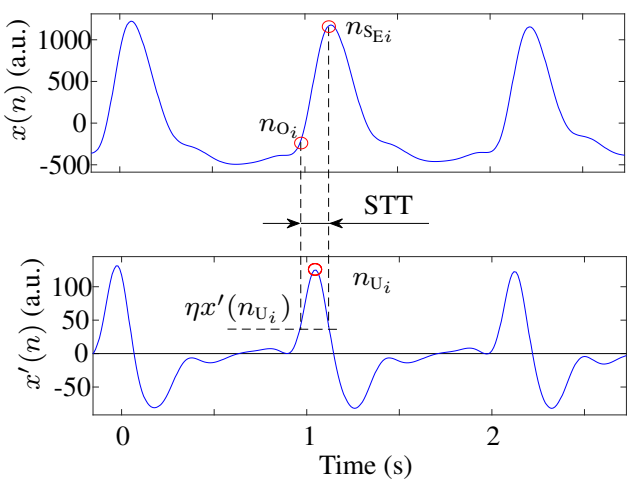

Figure 1. Example of $x(n)$ and $x^{\prime}(n)$ with definitions of $n_{\mathrm{U}_{i}}, n_{\mathrm{O}_{i}}, n_{\mathrm{SE}_{\mathrm{E} i}}$, and STT.

Note that none of the studied 5 series are evenly sampled as pulses occur non-uniformly in time. For each one of the series, a median-absolute-deviation-based outlier-rejection rule [3] was applied, and a 4-Hz evenly sampled version was obtained by cubic spline interpolation.

\subsection{Feature extraction}

For each 1-min fragment, the standard deviation (SD) of each one of the 5 PPG-derived series were used as features, representing the power of the oscillations. In addition, 3 features from the frequency domain were also extracted. In order to estimate the power spectral density (PSD) of each one of the 5 series, the Welch periodogram was applied using a Hamming window of $40 \mathrm{~s}$ and an overlap of $75 \%$. The power at low frequency band (LF, $[0.03 \mathrm{~Hz}, 0.15$ $\mathrm{Hz}])$, the power at high frequency band (HF, $[0.15 \mathrm{~Hz}, 0.4$ $\mathrm{Hz}]$ ), and its normalized version with respect to LF+HF (LFn) were computed by integrating the PSD and used as features. In this way, a total of 20 features were extracted from each 1-min fragment of PPG signal.

Artifactual segments were automatically detected and excluded from the analysis if any of the following criteria is fulfilled: 1) the segment contains an artifact according to the artifact detector described in [10], or 2) the quality of the segment is bad according to the pulse-to-pulse-intervalbased criteria described in [11].

\subsection{Classification}

The data set was divided into 2 groups: Training set and Test set. This division was performed ensuring that all the segments from each one of the subjects belongs to the same set, and trying to obtain subjects with similar number of OSA segments (less represented class) in both sets. In order to satisfy these criteria, subjects were sorted by their number of OSA segments. Subsequently, the selection was performed in an alternating way. Furthermore, in order to 
balance the groups at the training stage, the number of NB segments from each subject in the Training set was reduced to the number of OSA segments of that subject. This selection was performed based on k-means in order to obtain a good representation of the underlying distribution of the data. Table 1 shows details about Training and Test sets.

Table 1. Details about Training and Test sets.

\begin{tabular}{crr} 
& Training set & Test set \\
\hline \hline \# subjects & 13 & 13 \\
\# OSA segments & 286 & 367 \\
\# NB segments & $* 286$ & 3548 \\
\hline
\end{tabular}

*Note that Training set was balanced by k-means. A total of 3556 NB segments were available for the training set before the balancing.

A least squares support vector machine (LS-SVM) classifier with an RBF kernel [12] was used in this study. This classifier was chosen because it offered the best performance in a previous ECG-based study [1]. Training set was used for performing a feature selection by a forwardwrapper approach. Features were added consecutively maximizing the area under the receiver operating characteristic curve (AUC). As ANS regulation is different between sleep stages and particularly between rapid eye movement (REM) and non-REM stages [13], a subset of the data set was also analyzed, consisting of all segments not containing REM sleep stage.

\section{Results}

A total of 8 features were selected by the forwardwrapper approach: SD of PRV; LFn and HF of PAV; LF of PWV; SD and LF of pulse upslopes; and LFn and HF of STT. Table 2 shows accuracy (Acc), sensitivity (Se), specificity (Sp), positive predictive value (+PV), and AUC which were obtained when using the classifier applying the selected features as inputs to the Test set, excluding and not excluding the REM labeled segments. Figure 2 shows the obtained receiver operating characteristics curves.

Table 2. Performance of the classifier on the Test set.

\begin{tabular}{crr} 
& No REM exclusion & REM exclusion \\
\hline \hline Acc & $72.66 \%$ & $73.51 \%$ \\
Se & $73.81 \%$ & $71.99 \%$ \\
Sp & $72.55 \%$ & $73.65 \%$ \\
$+\mathrm{PV}$ & $21.12 \%$ & $20.73 \%$ \\
AUC & $82.12 \%$ & $79.68 \%$ \\
\hline \hline
\end{tabular}

\section{Discussion}

In this paper, the potential for discriminating OSA from NB was analyzed using 5 series which can be derived from

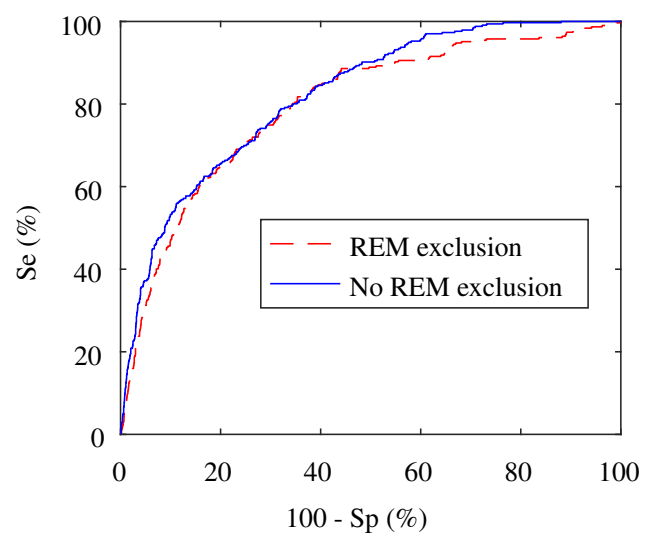

Figure 2. Receiver operating characteristics curve obtained with the Test set.

the PPG signal and which have been related to respiration: PRV, PAV, PWV, pulse upslope, and STT. For each series, 4 features related to its power were extracted: LF, HF, LFn, and SD. The 5 series are expected to be affected by both sympathetic modulations in low frequency, and by respiration, which is often in high frequency but may fall to low frequency. Thus, LFn could be interpreted as a sympathetic marker, and SD a sum of both effects.

All features were higher during OSA than during NB. This could be explained by a sympathetic activation in case of LF and LFn, by an intensification of respiratory effort in case of HF, and by any of the previous effects in case of SD. Increases of LF and LFn may be explained also by a fall of respiratory rate below $0.15 \mathrm{~Hz}$.

Training and Test sets were composed of segments from different subjects avoiding to obtain biased results due to possible subject overfitting. Furthermore, the number of OSA segments is much lower than the number of NB segments. In order to obtain a balanced training set, a kmeans-based selection of NB segments was performed ensuring that each one of the subjects of Training set contribute the same number of OSA segments as NB segments. In contrast, no balancing techniques were applied on Test set in order to obtain results from a real scenario.

The classifier obtained an AUC of $82.12 \%$, which is higher than other PPG-based methods in the literature such as [6] where an AUC of $72 \%$ was reported. Still, the obtained Acc was $72.66 \%$, similar to the $69 \%$ reported in [6]. This Acc is low for clinical diagnosis purposes but it is interesting for screening purposes taking into account the convenience of PPG signal for such devices. However, only OSA pulmonary events were analyzed, excluding from the analysis other pulmonary events with a high diagnostic relevance such as obstructive hypopnea and central and mixed apnea/hypopnea. One of the possible confounders are artifacts, as PPG signal is very affected by them and some of them may not be detected by 
the applied techniques. Furthermore, the obtained $+\mathrm{PV}$ is as low as $21.12 \%$ indicating a high number of false positives. These false positives could be explained by sympathetic activations during sleep which are not related to an apnea/hypopnea event, such as those occurring physiologically during REM sleep [13]. However, results did not improve when excluding the segments during REM sleep from the analysis. Similar Acc and +PV were obtained (73.51\% and $20.73 \%$, respectively), suggesting that REM sleep is not the only cause of those non-apnea/hypopnearelated sympathetic activations during sleep. Another possible explanation could be a low respiratory arousal threshold that makes arousal to be generated before the respiratory flow falls enough to be considered an apnea/hypopnea event according to the $\mathrm{AASM}_{12}$ criteria. Also, respiratory rate falling below $0.15 \mathrm{~Hz}$ during NB would increase LF and LFn and may produce a false positive. Information about respiratory rate, which can be extracted form the PPG signal, may help to improve + PV.

\section{Conclusions}

These results suggest that the studied features are promising for the discrimination of OSA from NB using only the PPG signal. Further studies must be elaborated to improve the $+\mathrm{PV}$, and to assess the performance when analyzing other pulmonary events such as obstructive hypopnea and central and mixed apnea/hypopnea.

\section{Acknowledgements}

This work is supported by: Agentschap voor Innovatie door Wetenschap en Technologie (IWT) under project SWT 150466 - OSA+. iMinds Medical Information Technologies: ICON HBC.2016.0167. European Research Council: The research leading to these results has received funding from the European Research Council under the European Union's Seventh Framework Programme (FP7/2007-2013) / ERC Advanced Grant: BIOTENSORS (n 339804). TIN2014-53567-R (MINECO, Spain), T96 (Government of Aragon and European Social Fund), CIBER-BBN (Instituto de Salud Carlos III and FEDER). This paper reflects only the authors' views and the Union is not liable for any use that may be made of the contained information. Carolina Varon is a postdoctoral fellow of the Research Foundation-Flanders (FWO). This project has received funding from the European Union's Framework Programme for Research and Innovation Horizon 2020 (2014-2020) under the Marie Skłodowska-Curie Grant Agreement No. 745755.

\section{References}

[1] Varon C, Caicedo A, Testelmans D, Buyse B, Van Huffel S. A novel algorithm for the automatic detection of sleep ap- nea from single-lead ECG. IEEE Trans Biomed Eng 2015; 62(9):2269-2278.

[2] Marín JM, Carrizo SJ, Vicente E, Agusti AGN. Longterm cardiovascular outcomes in men with obstructive sleep apnoea-hypopnoea with or without treatment with continuous positive airway pressure: an observational study. Lancet 2005;365:1046-1053.

[3] Lázaro J, Gil E, Bailón R, Mincholé A, Laguna P. Deriving respiration from photoplethysmographic pulse width. Med Biol Eng Comput 2013;51(1).

[4] Suzuki T, Kameyama K, Inoko Y, Tamura T. Development of a sleep apnea event detection method using photoplethysmography. In 32nd Annual International Conference of the IEEE EMBS. 2010; 5258-5261.

[5] Lázaro J, Gil E, Vergara JM, Laguna P. Pulse rate variability analysis for discrimination of sleep-apnea-related decreases in the amplitude fluctuations of PPG signal in children. IEEE J Biomed Health Inform 2014;18(1):240-246.

[6] Garde A, Dehkordi P, Ansermino JM, Dumont GA. Correntropy-based pulse rate variability analysis in children with sleep disordered breathing. Entropy 2017;19:282.

[7] Kahandoker AH, Karmakar CK, Penzel T, Gles M, Schoebel C, Palaniswami M. Estimating relative respiratory effort from features of photo-plethysmography signal. In 35th Annual International Conference of the IEEE EMBS. 2013; 6575-6578.

[8] Addison PS. Slope transit time (stt): A pulse transit time proxy requiring only a single signal fiducial point. IEEE Trans on Biomed Eng 2016;63(11):2441-2444.

[9] Berry RB, Budhiraja R, Gottlieb DJ et al. Rules for scoring respiratory events in sleep: Update of the 2007 AASM manual for the scoring of sleep and associated events: Deliberations of the sleep apnea definitions task force of the american academy of sleep medicine. J Clin Sleep Med 2012;8(5):597-619.

[10] Gil E, Vergara JM, Laguna P. Detection of decreases in the amplitude fluctuation of pulse photoplethysmography signal as indication of obstructive sleep apnea syndrome in children. Biomed Signal Process Control 2008;3:267-277.

[11] Orphanidou C, Bonnici T, Charlton P, Clifton D, Vallance D, Tarassenko L. Signal-quality indices for the electrocardiogram and photoplethysmogram: Derivation and applications to wireless monitoring. IEEE J Biomed Health Inform 2015;19(3):832-838.

[12] Suykens JAK, Van Gestel T, De Brabanter J, De Moor B, Vandewalle J. Least Squares Support Vector Machines. World Scientific, 2002.

[13] Somers VK, Dyken ME, Mark AL, Abboud FM. Sympathetic-nerve activity during sleep in normal subjects. N Engl J Med 1993;328(5):303-307.

Address for correspondence:

Jesús Lázaro

ESAT/STADIUS/KU Leuven

Kastelpark Arenberg 10, box 2446, 3001 Leuven, Belgium

jesus.lazaro@esat.kuleuven.be 